\title{
Computation of blocking probability for large circuit switched networks
}

\author{
Vyacheslav Abramov, Shuo Li, Meiqian Wang, Eric W. M. Wong, Senior Member, IEEE, Moshe Zukerman, \\ Fellow, IEEE
}

\begin{abstract}
The ever-growing Internet and the mounting evidence to the important role of circuit switching motivate the need for an accurate and scalable means for performance evaluation of large circuit switched networks. Previous work has shown that the Erlang Fixed Point Approximation (EFPA) achieves accurate results for such networks where the number of channels (circuits) per link is large. However, a conventional application of EFPA for large networks is computationally prohibitive. In cases where the EFPA solution is unattainable, we propose, in this paper, to use an asymptotic approximation, which we call A-EFPA, for the link blocking probability and demonstrate savings of many orders of magnitudes in computation time for blocking probability approximation in realistically sized networks with large number of circuits per link. We demonstrate for NSFNet and Internet 2 accurate calculations of the blocking probability using simulations, EFPA and A-EFPA, where each of these three methods is used for a different range of parameter values.
\end{abstract}

Index Terms-Erlang Fixed Point Approximation, Erlang B Formula, circuit switched networks, blocking probability.

\section{INTRODUCTION}

For several decades, numerous publications predicted and promoted an increasing role of circuit switching (CS) in the evolving ultra-broadband multi-service Internet. As early as 1988, O'Reilly [1] pointed out advantages of CS over packet switching that include easier provision of grade of service, simpler transport (which implies energy saving) and better suitability for multimedia services. CS attributes have given rise to dynamic CS-based network technologies and designs such as optical flow switching [2], and networks tailored to transmit large bursts from the Large Hadron Collider [3] and for inter-data center traffic [4].

Considering the renewed CS importance, and the exponential growth of the Internet, there is a need for scalable and accurate means to evaluate blocking probability in large CS networks. The Erlang Fixed Point Approximation (EFPA) has been considered the method of choice for this purpose since 1964 [5]. EFPA decouples the network into independent server groups (links), and considers the traffic on each link to follow a Poisson process independent traffic on other links. At each iteration of the EFPA algorithm, the blocking probability on each link is computed, where its offered traffic comprises all the end-to-end traffic streams that use that link minus the

V. Abramov is with the Centre for Advanced Internet Architectures, Swinburne University, Australia.

S. Li, M. Wang, E. W. M. Wong and M. Zukerman are with the Electronic Engineering Department, City University of Hong Kong, Hong Kong SAR.

The work described in this paper was supported by a grant from City University of Hong Kong [No. 9380044]. blocked traffic (which initially can be set to zero). Then, endto-end blocking probabilities on all links are recalculated and the process repeats itself until convergence is achieved.

The Poisson and independence assumptions introduce errors. However, Kelly [6] showed that for a CS network with fixed routing, EFPA yields an asymptotically exact solution for a large number of channels per trunk. Today's DWDM networks with nearly hundred wavelengths per optical fiber and hundreds optical fibers per cable yielding tens of thousands wavelength channels per cable. If a wavelength is further subdivided into hundreds of TDM channels, millions of channels per cable is a realistic scenario. Furthermore, the analysis of Kelly [6] applies to multiple classes of calls each requires a different number of channels and its performance result is insensitive to the shape of the holding time distribution.

Given that the results of [6] are applicable to today's networks, it is important to apply them in a way that the blocking probability is computable for realistic size networks. Using the conventional EFPA which repeatedly computes the link blocking probability by applying a recursive procedure to the Erlang-B formula, will be computationally infeasible for such networks with many channels per cable. In this paper, we consider an implementation of EFPA, which we call Asymptotic EFPA (A-EFPA) where the computation of the link blocking probability is performed using the asymptotic result for a large number of channels per cable which is essentially based on a fluid approximation. A-EFPA can achieve comparable accuracy to EFPA when the number of channels per cable is large, but it saves many orders of magnitudes computing time, so it can be used in the range where EFPA cannot. We demonstrate for NSFNet and Internet2 networks, that given today's computing power, simulations can be used in the range that EFPA is inaccurate, and now A-EFPA is shown to be accurate in the range where EFPA is computationally prohibitive. Accordingly, for both networks, very accurate blocking probability results are achievable for the full range of practical parameter values.

Henceforth, we use the terminology of [6]. In particular, we use the term circuit to describe the basic channel used for a connection (e.g. a wavelength channel) and the term link to refer to a trunk where many circuits are bundled together to provide transport between nodes.

\section{MODEL}

We consider a general CS network. The links between nodes are numbered by $j=1,2, \ldots, J$, and $C_{j}$ is the number of 
circuits in link $j$. The subscript is omitted if all links have the same number of circuits (then $C=C_{j}$ for all $j$ ). For convenience the notation $\mathcal{I}=\{1,2, \ldots, J\}$ is used in the sequel. A call on route $r$ uses $A_{j, r}$ circuits from link $j$. Let $\mathcal{R}$ be a set of possible routes. Assume that calls requesting route $r$ arrive as an independent Poisson process of rate $\mathrm{v}_{r}$. A call requesting route $r$ is blocked and lost if at least in one of the links $j, j \in \mathcal{I}$, in route $r$ there are less than $A_{j, r}$ circuits free. Otherwise, the call is admitted and simultaneously holds $A_{j, r}$ circuits from all links $j, j \in \mathcal{I}$, for the holding period of the call. Without loss of generality the call holding times are assumed to be independently and identically distributed with unit mean. Notice that, according to the analysis and asymptotic conditions of [6], the blocking probability at the limit is only a function of the offered load and number of circuits per link. Therefore, the arrival rate can be adjusted to compensate for non-unity holding times, and a call that requires 10 circuits is equivalent to 10 calls requiring one circuit, if the total offered load for each origin-destination (OD) pair is unchanged.

\section{A-EFPA ALGORITHM}

As in [6], we consider a sequence of networks indexed by $N$ where the number of circuits per link and the offered traffic per each OD pair grows linearly in $N$. For positive $x$ and integer $C$, denote $E(x, C)=\frac{x^{C} / C !}{\sum_{i=0}^{C} x^{i} / i !}$. It is proved in [6] that the system of equations

$$
x_{j}=E\left(\frac{1}{1-x_{j}} \sum_{r \in \mathcal{R}} A_{j, r} v_{r} \prod_{i=1}^{J}\left(1-x_{i}\right)^{A_{i, r}}, C_{j}\right), j \in \mathcal{J}
$$

has a unique solution in $(0,1)^{J}$. In Eq. (1), the blocking probability $x_{j}$ of link $j$ is obtained by Erlang-B formula based on this link capacity $C_{j}$ and the total offered load to that link (i.e. excluding traffic blocked on other links) of the OD pairs that use this link. EFPA solves (1) by fixed-point iterations. For (1), the following limit theorem for a series of CS networks is proved in [6].

Theorem 3.1: (Kelly [6]) Assume that the parameters $v_{r}(N)$ and $C_{j}(N),(r \in \mathcal{R}, j \in \mathcal{I})$, increase to infinity such that

$$
\lim _{N \rightarrow \infty} \frac{\mathrm{v}_{r}(N)}{N}=\lambda_{r}, \lim _{N \rightarrow \infty} \frac{C_{j}(N)}{N}=c_{j}
$$

Then,

$$
\lim _{N \rightarrow \infty} \prod_{j=1}^{J}\left[1-x_{j}(N)\right]^{A_{j, r}}=\prod_{j=1}^{J}\left(1-B_{j}\right)^{A_{j, r}},
$$

where $\mathbf{x}(N)=\left\{x_{1}(N), \ldots, x_{J}(N)\right\}$ is a series of the vectorvalued solutions of (1) indexed by $N$, and $B_{j}, j \in \mathcal{I}$, is the asymptotic blocking probability on link $j$.

According to Theorem 3.1, for large circuit-switched networks the blocking probabilities $B_{j}$ are supposed to be approximated by (1), in which the parameters $\mathrm{v}_{r}(N)$ and $C_{j}(N)$ are large. The further asymptotic analysis enables us to substantially simplify the calculations. As $N \rightarrow \infty$, for the function $E(x(N), C(N))$, in which $x(N) \asymp x^{*} N$ and $C(N) \asymp c^{*} N$, where $x^{*}$ is a positive real value and $c^{*}$ is a positive integer value, we have the following asymptotic expansion:

$$
E(x(N), C(N))= \begin{cases}1-\frac{c^{*}}{x^{*}}+o(1), & \text { if } c^{*} \leq x^{*}, \\ o(1), & \text { otherwise. }\end{cases}
$$

Henceforth, for two sequences $x_{n}$ and $y_{n}$ increasing to infinity $x_{n} \asymp y_{n}$ means $\lim _{n \rightarrow \infty} \frac{x_{n}}{y_{n}}=1$.

The difficulty of using asymptotic expansion (2) directly is that the expression

$$
\frac{1}{1-x_{j}} \sum_{r \in \mathcal{R}} A_{j, r} v_{r} \prod_{i=1}^{J}\left(1-x_{i}\right)^{A_{i, r}}
$$

contains unknowns $x_{j}, j=1,2, \ldots, J$, and, hence, we do not know whether or not the inequality

$$
\frac{1}{1-x_{j}(N)} \sum_{r \in \mathcal{R}} A_{j, r} v_{r}(N) \prod_{i=1}^{J}\left(1-x_{i}(N)\right)^{A_{i, r}} \geq C_{j}(N)
$$

is satisfied. Accordingly, if we ultimately use the term

$$
1-C_{j}(N)\left[\frac{1}{1-x_{j}(N)} \sum_{r \in \mathcal{R}} A_{j, r} v_{r}(N) \prod_{i=1}^{J}\left(1-x_{i}(N)\right)^{A_{i, r}}\right]^{-1}
$$

which is the asymptotic expansion of (2) applied to the righthand side of (1) under the asymptotic conditions of Theorem 3.1 , then we may arrive at biased values for the estimates of blocking probabilities (in the cases where terms (3) are negative). We use the term biased to refer to values that are not the right blocking probabilities (which cannot be negative).

Example Consider a simple CS network consisting of two links $a$ and $b$ with capacities $C_{a}$ and $C_{b}$, respectively, and assume that there are two types of calls: $a b$-calls that use a circuit in both links $a$ and $b$ and $b$-calls that use a circuit in link $b$ only. Denote by $v_{a b}$ the input rate of $a b$-calls and by $v_{b}$ the input rate of $b$-calls. For a series of networks assume $C_{a}(N)=C_{b}(N)=c N, v_{a b}(N)=v_{b}(N)=\lambda N$ and $c<\lambda$. These yield the following system of equations.

$$
\begin{gathered}
x_{a}=1-\frac{c}{\lambda\left(1-x_{b}\right)}, \\
x_{b}=1-\frac{c}{\lambda\left(1-x_{a}\right)+\lambda} .
\end{gathered}
$$

It is readily seen from this system of equations that $x_{a}<0$. Indeed, assume that $x_{a}>0$. Then, it follows from (4) that $x_{b}<1-\frac{c}{\lambda}$. From (5) we obtain the opposite inequality: $x_{b}>$ $1-\frac{c}{\lambda}$. This contradiction leads to the conclusion $x_{a} \leq 0$. Now substituting $x_{a}=0$ into (4) we obtain $x_{b}=1-\frac{c}{\lambda}$. The same substitution into (5) yields $x_{b}=1-\frac{c}{2 \lambda}$. Hence, $x_{a}$ cannot be equal to zero, and we arrive at $x_{a}<0$. This solution gives biased values for blocking probabilities. Negative $x_{a}$ value has to be set to zero, and $x_{b}$ has to be recalculated accordingly.

A-EFPA is based on decomposing the set $\mathcal{I}$ into three nonintersected subsets: $\mathcal{I}=\mathcal{I}^{-} \cup \mathcal{I}^{0} \cup \mathcal{I}^{+}$as described below.

1. Check the inequalities

$$
\sum_{r \in \mathcal{R}} A_{j, r} \lambda_{r}>c_{j}
$$

for all $j \in \mathcal{I}$. Then, the subset $\mathcal{g}^{0}$ characterizes the set of values $j$ for which (6) is not satisfied. Set $x_{j}=0$ for all $j \in \mathcal{g}^{0}$. By 
separating the subset of links $g^{0}$, we reduce the dimension of the original system of equations. The new system of equations is as follows:

$$
\begin{aligned}
& x_{j}=E\left(\frac{1}{1-x_{j}} \sum_{r \in \mathcal{R}} A_{j, r} \vee_{r} \prod_{i=1}^{J}\left(1-x_{i}\right)^{A_{i, r}}, C_{j}\right), \\
& j \in \mathcal{I} \backslash \mathcal{I}^{0} .
\end{aligned}
$$

2. Solve the system of equations, in which the right-hand side of (7) is replaced by the main term in the asymptotic expansion:

$$
x_{j}=1-c_{j}\left[\frac{1}{1-x_{j}} \sum_{r \in \mathcal{R}} A_{j, r} \lambda_{r} \prod_{i=1}^{J}\left(1-x_{i}\right)^{A_{i, r}}\right]^{-1}, \quad j \in \mathcal{J} \backslash \mathcal{g}^{0} .
$$

Let $\mathcal{g}^{-}$be the set of values $\mathcal{g} \backslash \mathcal{g}^{0}$ for which $x_{j} \leq 0$. Set $x_{j}=0$ for all $j \in \mathcal{I}^{-}$. Then, after eliminating the set of equations in which $j \in \mathcal{I}^{-}$, we obtain the new system of equations:

$$
\begin{gathered}
x_{j}=1-c_{j}\left[\frac{1}{1-x_{j}} \sum_{r \in \mathcal{R}} A_{j, r} \lambda_{r} \prod_{i=1}^{J}\left(1-x_{i}\right)^{A_{i, r}}\right]^{-1}, \\
j \in \mathcal{I}^{+}=\mathcal{J} \backslash\left(\mathcal{J}^{0} \cup \mathcal{I}^{-}\right) .
\end{gathered}
$$

3. Solve the system of equations (8). This is the final step, where we obtain only a positive solution. That positive solution together with the solutions that are set 0 in steps 1 and 2 give a full set of the asymptotic solution of the system of equations.

A-EFPA is essentially based on a reduction to a fluid model. If the traffic offered to a link is higher than its capacity, the difference between the offered traffic and capacity is the blocked traffic, and the ratio of the blocked traffic to the offered traffic is the blocking probability. Otherwise, the blocking probability is zero.

\section{NumericAl Results}

In this section, we compare the blocking probability and running time of EFPA and A-EFPA for NSFNet (13 nodes and 16 bidirectional links) and Internet2 (52 nodes and 60 bidirectional links) networks - see Fig. 1. For both networks, we choose all possible OD pairs with shortest path routing where a tie is broken randomly. Simulation results are also provided whenever they are achievable within reasonable time. All the results are obtained using MATLAB software executed on a desktop PC with Intel $\mathbb{R}$ Core $^{\mathrm{TM}} 2$ Quad @ $3 \mathrm{GHz}$ CPU, $4 \mathrm{GHz}$ RAM and 32-bit operating system.

For NSFNet, there are 156 OD pairs. For each OD pair, the offered load is set to be $0.025 C$, so that the total load offered to some of the links is larger than the capacity (note that in the NSFNet example, some links serve almost 100 OD pairs). Fig. 2a illustrates that only when the capacity is sufficiently large, the A-EFPA results are very close to those of EFPA. For $C \geq 20,000$, the relative discrepancy is less than $0.2 \%$. Simulations confirm that the accuracy of EFPA increases with increasing number of circuits per link. Confidence intervals are too small to be seen in the plots, but for the results presented, the radius of the $95 \%$ confidence interval based on Student's $\mathrm{t}$-distribution is less than $1.5 \%$ of the mean.
Table I provides the running time used to calculate the blocking probability in NSFNet for different $C$ values. Observe that for $C=20000$, A-EFPA saves $99.9999 \%$ of the time used by the EFPA, and achieves similar accuracy.

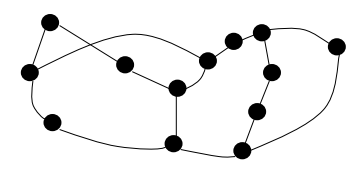

(a) NSFNet

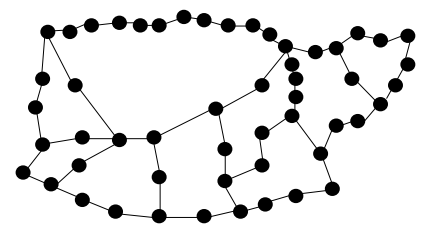

(b) Internet 2
Fig. 1: NSFNet and Internet2 topologies.

TABLE I: Comparison of the times used by EFPA and AEFPA to calculate the blocking probabilities in NSFNet.

\begin{tabular}{|c|c|c|}
\hline \hline Calculation task & $\begin{array}{c}\text { Running time of } \\
\text { EFPA in seconds }\end{array}$ & $\begin{array}{c}\text { Running time of } \\
\text { A-EFPA in seconds }\end{array}$ \\
\hline $\begin{array}{c}\text { Blocking probabilities in } \\
\text { one link and } C=20000\end{array}$ & 14.94 & 0.000001 \\
\hline $\begin{array}{c}\text { Blocking probability of the } \\
\text { whole network and } C=2000\end{array}$ & 5.91 & 0.068 \\
\hline $\begin{array}{c}\text { Blocking probability of the } \\
\text { whole network and } C=20000\end{array}$ & 1800.63 & 0.069 \\
\hline \hline
\end{tabular}

It is intuitively clear that if the offered load is sufficiently small, the offered load on all links will be less than their capacities, so the link blocking probabilities calculated by AEFPA are all 0, thus the network blocking probability is also 0 . In such a scenario, according to the theory, as $C \rightarrow \infty$, the exact blocking probability and the blocking probability predicted by EFPA, both will also approach zero. However for fixed $C$, there will be some error for using A-EFPA. In Table II, we illustrate the effect of traffic load on the accuracy of A-EFPA for NSFNet in the case of $C=20,000$. In the first row, we illustrate the case of zero blocking predicted by A-EFPA where although the relative error is $100 \%$, the absolute error is small and will be further reduced as $C$ increases. Then, we observe improved accuracy of A-EFPA as the traffic increases.

TABLE II: Effect of offered load on A-EFPA.

\begin{tabular}{|c|c|c|c|c|}
\hline \hline \multirow{2}{*}{ Offered load } & Blocking probability & \multirow{2}{*}{ Difference } & \multirow{2}{*}{ Relative error } \\
\cline { 2 - 3 } & EFPA & A-EFPA & & \\
\hline 0.0205 & $9.9 \times 10^{-10}$ & 0 & $9.9 \times 10^{-10}$ & $100 \%$ \\
\hline 0.022 & 0.0103034 & 0.0099068 & 0.0003966 & $3.8 \%$ \\
\hline 0.025 & 0.0449448 & 0.0448718 & 0.0000730 & $0.16 \%$ \\
\hline 0.035 & 0.1786133 & 0.1785714 & 0.0000410 & $0.023 \%$ \\
\hline \hline
\end{tabular}

For Internet2, for each OD pair, the offered load is set to be $0.12 \%$ of $C$, so that the total load offered to some links is larger than their capacities (note that in our Internet2 example some links serve around a thousand OD pairs). The blocking probabilities for Internet 2 evaluated by EFPA and A-EFPA are shown in Fig. 2b. As for NSFNet, the results obtained by A-EFPA are very close to those of EFPA when 
(a) NSFNet
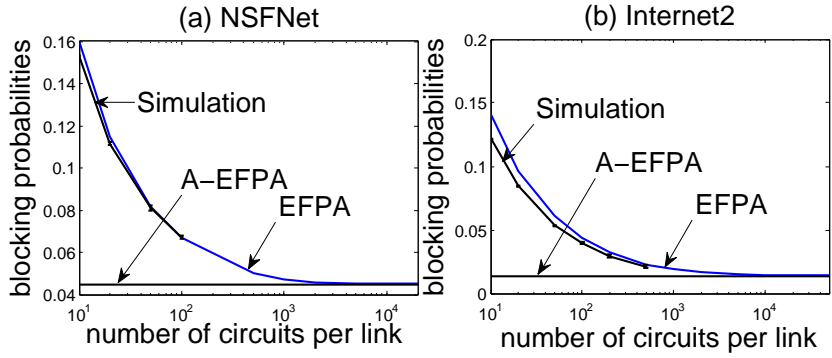

Fig. 2: Blocking probabilities in NSFNet and Internet2 evaluated by EFPA and A-EFPA.

the capacity is large. When the number of circuits per link reaches $C=50000$, the relative discrepancy (vs. EFPA) of the blocking probability calculated by A-EFPA is $1.0 \%$. However, comparing the time used by the two algorithms shown in Table IV, we observe that A-EFPA can save $99.9999 \%$ of the time. The trends and behaviors of the results presented for Internet 2 are consistent with the results provided for NSFNet. The limitation of simulations motivates using EFPA or AEFPA which are asymptotically exact as the number of circuits per link increases. Confidence intervals are again unnoticeable in the plots, but the radius of the $95 \%$ confidence interval based on Student's t-distribution is always less than $1.5 \%$ of the mean result. EFPA overestimates the blocking probability obtained by simulation because under fixed routing, traffic is smoothed out along an end-to-end path which gives lower loss than estimated by EFPA. This overestimation is more prominent in Internet 2 than in NSFNet because the average path in Internet 2 is longer.

TABLE III: Comparison of the blocking probabilities calculated by EFPA and A-EFPA for NSFNet and Internet2.

\begin{tabular}{|c|c|c|}
\hline \hline & EFPA & A-EFPA \\
\hline Blocking probability for & 0.04494 & 0.04487 \\
NSFNet with $C=20000$ & & \\
\hline Blocking probability for & 0.01417 & 0.01383 \\
Internet2 with $C=20000$ & & \\
\hline Blocking probability for & 0.01397 & 0.01383 \\
Internet2 with $C=50000$ & & \\
\hline \hline
\end{tabular}

TABLE IV: Comparison of the times used by EFPA and AEFPA to calculate the blocking probabilities in Internet2.

\begin{tabular}{|c|c|c|}
\hline \hline Calculation task & $\begin{array}{c}\text { Running time of } \\
\text { EFPA in seconds }\end{array}$ & $\begin{array}{c}\text { Running time of } \\
\text { A-EFPA in seconds }\end{array}$ \\
\hline $\begin{array}{c}\text { Blocking probability of the } \\
\text { whole network and } C=2000\end{array}$ & 66.68 & 0.72 \\
\hline $\begin{array}{c}\text { Blocking probability of the } \\
\text { whole network and } C=50000\end{array}$ & 62501.81 & 1.27 \\
\hline \hline
\end{tabular}

Blocking probability results for NSFNet with multirate traffic are presented in Fig. 3. There are two classes of calls in each OD pair. A Class 1 call requires a single channel endto-end and a Class 2 call requires five channels end-to-end. The offered load to each class in each OD pair is $0.015 C$. (a) Class 1
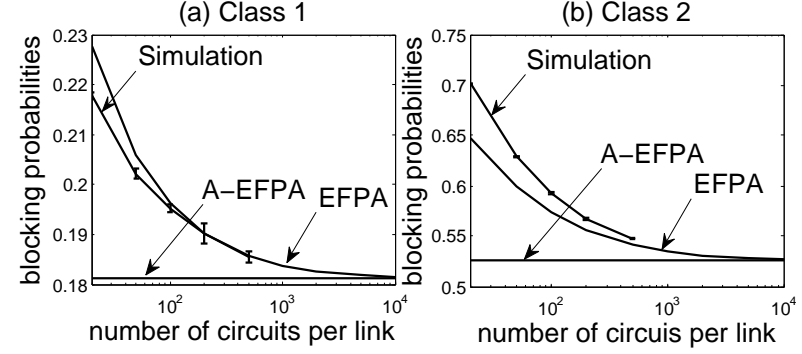

Fig. 3: Blocking probabilities in 13-node NSFNet evaluated by EFPA, A-EFPA and simulation with two classes of traffic.

The results presented exhibit similar trends and behavior as the results presented for NSFNet with single class of calls.

We observe that for Class 1 calls, EFPA overestimates the blocking probability as in the single class case due to the smoothing effect along the path. However, for Class 2 traffic EFPA underestimates the blocking probability because it assumes Poisson arrivals of individual circuit demands and ignores the larger variance introduced by the 5-circuit batch demands. These dominate the path smoothing effect, so the overall variance is higher than that assumed by EFPA.

In all the results presented here, we observe that the simulation curve meets (or becomes very close to) the EFPA curve, significantly before the EFPA and A-EFPA curves are very close. These indicate that given today's computing power, we are able using simulations to reach the point in terms of number of circuits per link, where EFPA is accurate, then EFPA can provide accurate results until A-EFPA becomes accurate, which in turn can guarantee accuracy for far larger values of circuits per link.

\section{CONCLUSION}

We have considered A-EFPA and EFPA implementation for CS networks with fixed routing based on asymptotic link blocking probability derivation. From the numerical results obtained using NSFNet and Internet 2 networks, we observe that when link capacity is large, A-EFPA results are very close to those of EFPA, but A-EFPA saves approximately $99.9999 \%$ of the computing time. We have demonstrated very accurate calculations of the blocking probability using simulations, EFPA and A-EFPA. Complementing each other, these three methods are used for different ranges of parameter values.

\section{REFERENCES}

[1] P. O'Reilly, "The case for circuit switching in future wide bandwidth networks," in Proc. IEEE ICC' 88, vol. 2, Jun. 1988, pp. 899-904.

[2] G. Weichenberg, V. Chan, and M. Medard, "Design and analysis of optical flow-switched networks," IEEE/OSA Journal of Optical Communications and Networking, vol. 1, no. 3, pp. B81-B97, Aug. 2009.

[3] A. Barczyk, "World-wide networking for LHC data processing," in Proc. NFOEC, Los Angeles, Mar. 2012, pp. 321-329, paper NTu1E.1.

[4] A. Mahimkar, A. Chiu, R. Doverspike, M. D. Feuer, P. Magill, E. Mavrogiorgis, J. Pastor, S. L. Woodward, and J. Yates, "Bandwidth on demand for inter-data center communication," in Proc. ACM HotNets '11, New York, NY, USA, 2011, pp. 24:1-24:6.

[5] R. B. Cooper and S. S. Katz, "Analysis of alternate routing networks with account taken of the nonrandomness of overflow traffic," Bell Telephone Lab, Tech. Rep., 1964, memo.

[6] F. P. Kelly, "Blocking probabilities in large circuit-switched networks," Adv. in Appl. Probab., vol. 18, no. 2, pp. 473-505, Jun. 1986. 